\title{
Fibrin Packing Arrangements Inferred from Crystal Structures of Fragments D and Double-D \\ Complexed with Synthetic Peptide Knobs -Packing Arrangements in Fibrin-
}

\author{
Russell F. DOOLITTLE*
}

\begin{abstract}
Recent X-ray crystallography studies on fragments D and DD from fibrinogen and fibrin have revealed the nature of the interaction at the ends of the individual fibrin units. Additionally, cocrystallization of fragments with synthetic peptides patterned on the sequences exposed by the thrombin-catalyzed removal of the fibrinopeptides has provided the details of knob-hole interactions. Interestingly, fragments complexed with the synthetic peptide corresponding to the B knob (GHRPam) exhibit a large conformational change in the presence of the ligand, adding to the intrigue of the still not well understood role of fibrinopeptide B removal.
\end{abstract}

Key words : fibrinogen, fibrin, crystal structure, fragment D

\section{Introduction}

The principal aim of structural studies on fibrinogen and its fragments is to understand the details of how the units pack together in fibrin. The recent publication of several high resolution crystal structures of major fragments from fibrinogen and fibrin has gone a long way towards meeting that goal ${ }^{1 \sim 6)}$. Some aspects remain stubbornly unresolved, however. In this lecture (paper) I review some of the features that seem well settled, as well as those that need more work. In particular, I will dwell on the consequences of fibrinopeptide $B$ removal and the exposure of the "B knob".

It has long been a part of the conventional wisdom that the removal of the fibrinope- ptide A leads to the formation of intermediate protofibrils. These are formed by a staggered, half-molecule overlap, the end-result of which is a two-molecule thick, end-to-end, non-covalent oligomer. It is also generally accepted that the subsequent release of the fibrinopeptide $B$ from the units in these oligomers allows the lateral association needed to form the thick fibers that constitute natural clots $^{7}$. These conclusions were reached well before there was any structural information available about the relative locations of the sites involved. Eventually, it became clear that it was the exposure of centrally located Gly-Pro-Arg "knobs” fitting into "holes" on the ends of the fibrin units, themselves situated on the $\gamma$-chain carboxyl terminal domains, that gives rise to the for-

\footnotetext{
* Center for Molecular Genetics, University California, San Diego〔La Jolla, CA 92093-0634, USA〕

Fax : (858) 534-4985, e-mail :rdoolittle@usd.edu
} 
mation of the intermediate protofibrils. A tremendous body of biochemical evidence was accumulated to this end, including the identification of many variant human fibrinogens with amino acid substitutions in the critical areas ${ }^{8}$, as well as ligand-binding studies with synthetic peptides ${ }^{910)}$ and the photo-affinity labeling of $\boldsymbol{\gamma}$-chain residue Tyr 363 by Gly-Pro-Arg derivatives ${ }^{11212}$.

The evidence for the role of the "B knob", which begins with the sequence Gly-His-Arg, has been less direct. For example, the location or even the existence of a corresponding "hole" for B knobs has never been suggested on the basis of variant fibrinogens, nor was it possible to find such a site by photo-affinity labeling. And although synthetic peptides beginning with the sequence Gly-Pro-Arg are effective inhibitors of fibrin polymerization, Gly-His-Arg peptides are not ${ }^{910}$. Remarkably, Gly-His-Arg-Pro peptides actually accelerate fibrin formation ${ }^{9}$. In a similar vein, Gly-Pro-Arg-Pro affinity columns are very useful for preparing fibrinogen or fragment D, but Gly-His-Arg-Pro columns are wholly ineffective. It is also well known that fibrin can be formed by the removal of fibrinopeptide A only, as can be demonstrated with various snake venom enzymes. Nonetheless, such fibrin is not of the same quality as fibrin generated by the thrombin-catalyzed removal of both fibrinopeptides $\mathrm{A}$ and $\mathrm{B}$, and the physiological role of removing the latter must be significant.

The crystal structures of fragments D and double-D from fibrin have now shed some light on these enigmatic observations, partly by uncovering the exact locations of the holes, and partly by demonstrating a remarkable conformational change that is involved
Table 1 X-Ray Structures of Fragments D and $\mathrm{DD}$

Expected from Biochemistry and EM Coiled-coil boundaries Homologous $\beta$ and $\gamma$ carboxyl domains $\gamma$-chain calcium binding site Affinity-labeled Tyr-363 Locations of numerous variants

Some Unexpected Findings

Abrupt reversal of $\alpha$ chain

Nature of D-D interface

Location of B-hole

Calcium binding by $\beta$ chain

Conformational change at B-hole

Inaccessibility of $\alpha 151 \sim 157$ (t-PA activation)

Major Disappointment

$\gamma-\gamma$ Crosslinks not visible

with the formation of the $\beta$-chain sites (Table 1).

\section{Protofibril Formation}

Consider first the Gly-Pro-Arg "knob-hole" interaction and the end-to-end interaction which it encourages. The crystal structure of crosslinked DD fragments ${ }^{3}$ showed that the $\boldsymbol{\gamma}$-chain holes on abutting molecules are only about $30 \AA$ apart, about what would be expected if the two A knobs from another fibrinogen molecule were holding them together (Fig. 1). To be more precise, the $\mathrm{C} \alpha$ carbons of the respective proline residues at positions 4 of the bound peptides are $26 \AA$ apart. In native fibrinogen, the two $\alpha$ chains are held togther by a disulfide bond between the two cysteine residues at position $28^{13}$. As such, it was a simple matter to model in the missing residues between Arg-19 and Cys-28 to form a bridge between knobs.

We also synthesized the 12-residue peptide GPRVVERHQSAC that corresponds to this segment $(\alpha 17 \sim 28$ stretches from the end of the A knob to the disulfide bond) and oxid- 
Table 2 Fragments D and Related Structures Determined As of June, 1999

$\begin{array}{lll}\text { fragment D (high calcium) } & 2.9 \AA & \text { Spraggon et al, 1997 (Ref. 3) } \\ \text { double-GPRPam } & 2.9 \AA & \text { Spraggon et al, 1997 (Ref. 3) } \\ \text { double-D+GPRPam +GHRPam } & 2.3 \AA & \text { Everse et al, 1998 (Ref. 4) } \\ \text { double-D+GHRPam } & 2.7 \AA & \text { Everse et al, 1999 (Ref. 6) } \\ \text { fragment D+GHRPam } & 2.5 \AA & \text { Everse et al, 1999 (Ref. 6) } \\ \text { double-D, no ligands } & 3.0 \AA & \text { Everse et al, 1999 (Ref. 6) } \\ \text { fragment D (2.5 mM calcium) } & 3.0 \AA & \text { (Doolittle et al, unpublished) } \\ \text { double-D+bis-dodecamer } & 2.5 \AA & \text { (Doolittle et al, unpublished) } \\ \text { lamprey D+GHRPam } & 2.9 \AA & \text { (Doolittle et al, unpublished) } \\ \alpha \mathrm{C}^{\prime} \text { (recombinant) } & 2.1 \AA & \text { Spraggon et al, 1998 (Ref. 5) }\end{array}$

ized it to form the bis-dodecamer. The crystal structure of double-D complexed with this 24residue peptide was determined (Table 2). As it happens, the electron density of the peptide was only clear for the first five to seven residues on either side, including the bound Gly-Pro-Arg termini. The remainder of the peptide loop is not close bound to the DD fragment and is apparently mobile. This is an equivalent situation to that which occurs on the opposite side of the DD interface, where the crosslinked carboxyl-terminal segments of the $\gamma$ chains could not be visualized either, and for much the same reason (Fig. 2). It must be concluded from these observations that not only are the end-to-end connections between fibrin units loose and mobile, but so are the interactions between $\mathrm{D}$ and $\mathrm{E}$, the only tight attachments being the knob-hole interactions themselves.

A priori, there was some reason to think such a situation might exist ; scanning differential calorimetry experiments had long ago shown that there is no significant difference in the melting curves of the $\mathrm{D}$ and $\mathrm{E}$ domains in fibrin compared with fibrinogen ${ }^{14}$. If the association were intimate, it might have been expected that the melting curves would have been co-influenced, as occurs in the complexes of other interacting proteins. As one description put $\mathrm{it}^{15)}$, "the fibrin units are held together like dancers at arm's length rather than being nestled together cheek-tocheek".

There is another aspect to the interaction that was not anticpated. The nature of the interface between the abutting ends of the fibrin units is not tight; there are no obvious electrostatic interactions and hardly any hydrogen bonds ${ }^{3}$. There looks to be a layer of solvent between the complementary surfaces. At first glance this situation seems quite appropriate, since it would not be advantageous to have end-to-end association of fibrinogen molecules in advance of fibrinopeptide removal. Still, it is somewhat disconcerting that all of the crystals involving $\mathrm{D}$ (and of course DD) (Table 2) pack end-toend in very similar ways, as though this is a naturally stable situation. The same packing interaction apparently occurred in crystals of a modified bovine fibrinogen ${ }^{16)}$, and we have also recently found it in crystals of native chicken fibrinogen ${ }^{17}$. The only exception, and it may be significant, is that this interaction was not observed in the crystals of the isolated $\gamma \mathrm{C}$ domain ${ }^{1)}$. This might imply that the presence of the $\beta \mathrm{C}$ domain has an influence on the packing properties of the $\gamma \mathrm{C}$ domain. It could be a negative influence, of course, simply preventing the kind of packing that occurs in the recombinant $\gamma \mathrm{C}$ 


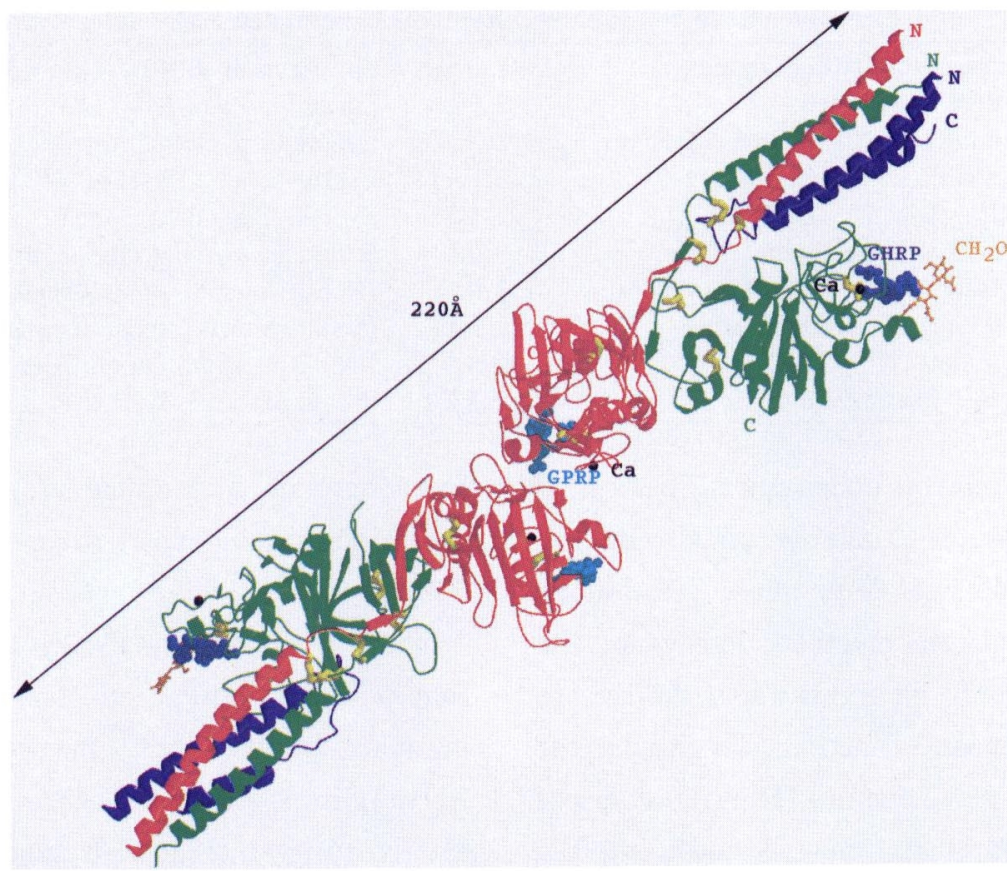

Fig. 1 Ribbon structure of double-D from cross-linked human fibrin with two bound ligands. GPRP, Gly-Pro-Arg knobs in $\gamma$-chain holes;GHRP, Gly-His-Arg knobs in $\beta$-chain holes. The monomeric fragment $\mathrm{D}$ units are cross-linked near their $\gamma$-chain carboxy-termini. (from Ref. 4).

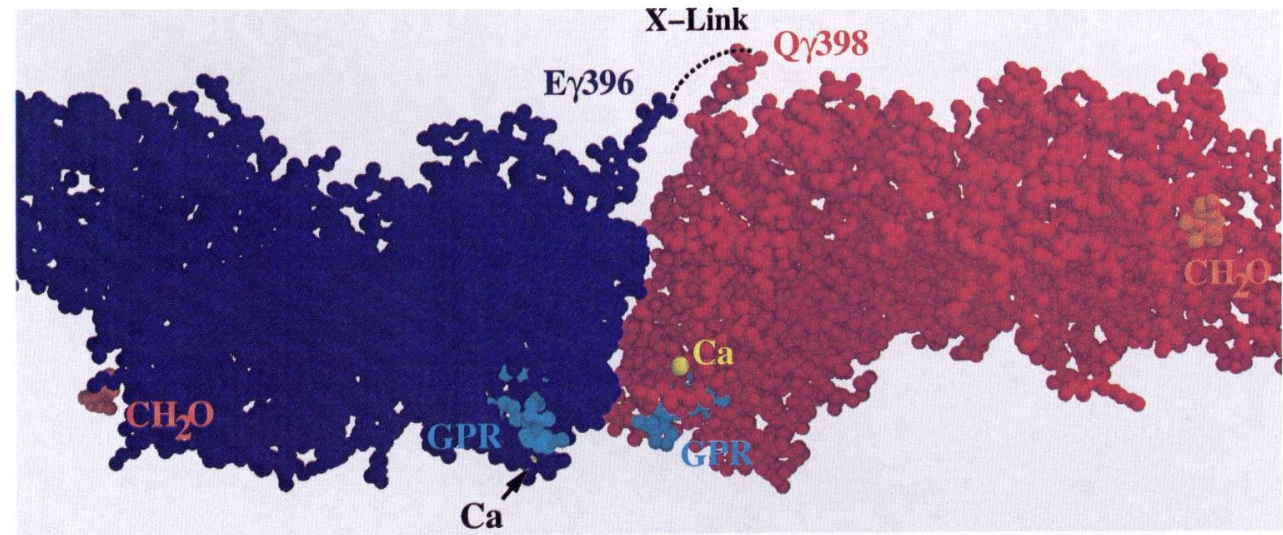

Fig. 2 Interfacial abutment of terminal units in fibrin showing relative locations of GPRP knobs, tightly bound calcium, and $\beta$-chain carbohydrate clusters. The $\boldsymbol{\gamma}-\boldsymbol{\gamma}$ crosslink is denoted by dashed line (from Ref. 3).

domain. I will build on the point later.

For the moment, however, our interpretation is that the strength of a single A knob bound to a $\gamma$-chain hole is not sufficient to hold the initiating system together. Rather, the end-to-end interaction likely occurs first, and the association is subsequently stabilized by the introduction of the two $\mathrm{A}$ knobs into their respective holes, the pinioned product being thermodynamically sturdy. The impor- 
tance of the order of these events is that it allows the knobs to be tethered to the central domain in a flexible way; they are not initally fixed in space in the same orientation that ultimately exists in the protofibril.

Summing to this point, the first step of fibrin formation involves a natural association at the DD-interface between two abutting fibrinogen molecules pinned together on one side by the two A knobs of another fibrinogen molecule. There is reason to believe that the end-to-end interaction occurs in advance of the pinioning, and that the flexible knobs find their way into a predetermined orientation. The union is further cemented by the action of factor XIII-induced crosslinks between the carboxyl-terminal segments of $\gamma$ chains located diametrically opposite to the knob-hole sites. Both of these interactions are loose enough that there must be flexibility in the protofibril chain.

\section{Association of Protfibrils}

Turning now to the involvement of the $\mathrm{B}$ knob, the crystal structures of fragments D and DD revealed that the $\beta$ chain has a "hole" at the same place as does the homologous $\gamma$ chain, but it is aimed in quite the opposite direction(Fig. 1). Indeed, it opens on the side of the $\beta C$ domain facing back toward the center of the fibrinogen molecule. Its location offers a possible explanation of why GHRP-affinity columns don't bind fibrinogen or fragment D. Thus, the arms in such columns may not have been long enough to allow the attached knobs to reach the somewhat hindered holes. Beyond that, its location apparently precludes a situation whereby a pair of B knobs from a single fibrinogen molecule could pin together two $\beta$ domains in the same way that a pair of A knobs holds together two $\gamma$ domains. In this regard, the $\beta$-chain holes on the two halves of a single fibrinogen molecule are approximately $300 \AA$ apart The two $\beta$-chain holes in a DD fragment are only half that distance, but still a long way apart.

The B knobs, in contrast to the A knobs which are held close to each other by the disulfide connection between the cysteines at position $\alpha 28$ mentioned above, must be extended from the parent molecule at an obtuse angle capable of accommodating the spiraling fiber. It should be noted that the $\mathrm{B}$ knobs (res. 15 17 of the $\beta$ chain) are approximately 50 residues away from a disulfide connection between the $\beta$ and $\alpha$ chains, whereas the A knobs (res. 17 19 of the $\alpha$ chain) are only 20 residues away from that same disulfide. It seems most reasonable that a single knob at the end of an extended arm is contributed by another fibrinogen molecule. The question is, where is that molecule? For steric and distance reasons, it is unlikely to emanate from one of the other molecules in the same protofibril. It is much more reasonable to expect it to come from another protofibril. Moreover, the expectation would be that the other $\mathrm{B}$ knob from that same molecule would extend to still a third protofibril. Such a mechanism would allow protofibrils to be continuously wound around in a spiral fashion to yield the mature fibrin.

However, in order to accommodate such an encircling extension, the protofibrils would have to be in exact register. It is well known that fibrin exhibits a well defined periodicity reflecting the half-molecule overlap $(230 \AA)$. If the $\mathrm{B}$ knobs exhibit the same 
(or greater) flexibility we have attributed to the A knobs, how could this perfect registration of protofibrils occur? If our reasoning about an interfacial association having to preceed the involvement of $\mathrm{A}$ knobs is correct, shouldn't we be expecting a similar precondition for the particpation of B knobs? The possibility of a $\beta-\beta$ interaction equivalent to the $\gamma-\gamma$ end-to-end interaction presents itself as a possibility for lateral association. Modeling studies may be able to resolve the matter.

\section{A Conformational Change}

It wasn't until we had solved the structure of a DD fragment with bound GHRPam that we realized that the $\beta$-chain hole was not fully formed in the absence of the ligand ${ }^{4}$. In this regard, there is a big swing of sidechains $\beta$ Glu 397 and $\beta$ Asp 398 from a position where they are pinned back against coiled coil by a calcium bridge ${ }^{6}$. Indeed, the conformational change may be the basis for the acceleration of fibrin formation in the presence of the GHRP-ligands ${ }^{9}$. The incomplete site may insure that knobs don't inadvertently bind to this site until protofibril formation has been completed. It may also contribute to the exposure of the $\beta$-chain face that is homologous to the region of $\gamma$ chains that provide the end-to-end interaction, thereby providing the rigid feature that keeps the protofibrils in exact register.

There are other possibilities, of course, and other aspects to consider. For example, a model of fibrin formation has been proposed that invokes the association of $\alpha \mathrm{C}$ domains ${ }^{18) 19}$. It is reasonable to suppose a role for these domains in fibrin formation, if only because it is well known that under native conditions $\alpha$ chains are slowly crosslinked through the action of factor XIII ${ }^{20)}$. It is far from certain that $\alpha \mathrm{C}$ domains are what lead to the association of the protofibrils, however, and if they are, we must then ask what is the role of the B knobs and their holes? It is also reasonable to suppose, as has been implied by this model, that there may be some kind of interaction between the fibrinopeptides $\mathrm{B}$ and the $\alpha \mathrm{C}$ domains, and that removal of the fibrinopeptides $B$ allows a relocation of the $\alpha \mathrm{C}$ domains from some kind of intramolecular association to an intermolecular one ${ }^{18) 19}$. Further studies and a crystal structure of native fibrinogen may resolve the issue.

\section{The Lamprey Paradox}

More than 35 years ago, it was found that lamprey fibrinogen can be clotted by the exclusive removal of its fibrinopeptide $\mathrm{B}^{21222)}$. These results have been steadfastly ignored by the blood clotting community, inspite of the fact that the lamprey fibrinogen-fibrin system closely resembles its mammalian counterpart, including its sensitivity to GlyPro-Arg inhibitors. To reinforce the point, we have determined the crystal structure of lamprey fragment $\mathrm{D}$ (Table 1) and found all its features to resemble the human fragment.

The lamprey fibrinopeptide $B$ results have always been puzzling. How can exposure of only the B knob result in clotting? The puzzle became even more intriguing when the locations of the $\beta$-chain holes were determined. The initial interactions must still be end-toend, because, in the presence of factor XIII, $\gamma \cdot \gamma$ dimers appear at the same relative rates. 
My feeling is that the B knobs, like the A knobs, are flexibly tethered and wander in space until they find appropriate holes. We know now from the crystal studies that $B$ knobs can fit into the $\gamma$-chain holes when there are no competing $\mathrm{A} \mathrm{knobs}^{4}$. This is exactly the situation that occurs when the fibrinopeptides B are removed but not the fibrinopeptides A. Modeling studies may be able to show whether there is sufficient freedom and space under such circumstances for $\mathrm{B}$ knobs to fit into the holes ordinarily occupied by the A knobs, thereby pinning together two molecules associated at the $\gamma-\gamma$ interface, and which would thereby be crosslinked by factor XIII in the usual way.

\section{References}

1) Yee VC, Pratt KP, Côté HCF, LeTrong I, Chung DW, Davie EW, Stenkamp RE, Teller DC. Crystal structure of a $30 \mathrm{kDa} C$-terminal fragment from the $\gamma$ chain of human fibrinogen. Structure 5 : 125-138, 1997.

2) Pratt KP, Côté, HCF, Chung DW, Stenkamp RE, Davie EW. The fibrin polymerization pocket: three-dimensional structure of a $30-\mathrm{kDa}$ C-terminal $\gamma$ chain fragment complexed with the peptide Gly-Pro-Arg-Pro. Proc Natl Acad Sci USA 94 : 7176-7181, 1997.

3) Spraggon G, Everse SJ, Doolittle RF. Crystal structures of fragment $\mathrm{D}$ from human fibrinogen and its crosslinked counterpart from fibrin. Nature 389 : 455-462, 1997.

4) Everse SJ, Spraggon G, Veerapandian L, Riley M, Doolittle RF. Crystal structure of fragment double-D from human fibrin with two different bound ligands. Biochemistry 37 : 8637-8642, 1998.

5) Spraggon G, Applegate D, Everse SJ, Zhang J-Z, Veerapandian L, Redman C, Doolittle RF, Grieninger G. Crystal structure of a recombinant extended $\alpha_{\mathrm{E}} \mathrm{C}$ domain from human fibrinogen-
420. Proc Natl Acad Sci USA 95 : 1-9, 1998.

6) Everse SJ, Spraggon G, Veerapandian L, Doolittle RF. Conformational changes in fragments $D$ and double-D from human fibrin (ogen) upon binding the peptide ligand Gly-His-Arg-Proamide. Biochemistry 38 :2941-2946, 1999.

7) Laurent TC, Blombäck, B. On the significance of the release of two different peptides from fibrinogen during clotting. Acta Chem Scand 12 : 1875-1977, 1958.

8) Matsuda M. The structure-function relationship of hereditary dysfibrinogens. Intern $\mathrm{J}$ Hematol 64 : 167-179, 1996.

9) Laudano AP, Doolittle RF. Synthetic peptide derivatives that bind to fibrinogen and prevent the polymerization of fibrin monomers. Proc Natl Acad Sci USA 75 : 3085-3089, 1978.

10) Laudano AP, Doolittle RF. Studies on synthetic peptides that bind to fibrinogen and prevent fibrin polymerization. Structural requirements, number of binding sites, and species differences. Biochemistry 19 : 1013-1019, 1980.

11) Shimizu A, Nagel G, Doolittle RF. Photoaffinity labeling of the primary fibrin polymerization site : Isolation and characterization of a labeled cyanogen bromide fragment corresponding to $\gamma$ chain residues 337-379. Proc Natl Acad Sci USA 89 : 2287-2892, 1992.

12) Yamazumi K, Doolittle RF. Photoaffinity labeling of the primary fibrin polymerization site: Localization of the label to $\gamma$-chain Tyr-363. Proc Natl Acad Sci USA 89 : 2893-2896, 1992.

13) Blombäck $B$ : Selectional trend in the structure of fibrinogen of different species, in Schffeniels $\mathrm{E}$ (ed) : Biochemical Evolution and the Origin of Life, North Holland Publishing Co, Amsterdam, 1971, 112-129.

14) Donavan JW, Mihalyi E. Conformation of fibrinogen : calorimetric evidence for a three-nodular structure. Proc. Natl. Acad. Sci. USA 71 : 4125-4128, 1974.

15) Doolittle RF : Fibrinogen and Fibrin, in Bloom AL, Thromas DP (eds): Haemostasis and Thrombosis, $2^{\text {nd }}$ ed. Churchill Livingstone. Edin- 
burgh, 1987.

16) Rao SPS, Poojary D, Elliot BW, Melanson LA, Oriel B, Cohen C. Fibrinogen structure in projection at $18 \AA$ resolution. electron density by $\mathrm{co}^{-}$ ordinated cryo-electron microscopy and X-ray crystallography. J Mol Biol 222 : 89-98, 1991.

17) Yang $Z$, Mochalkin I, Veerapandian L, Riley M, Doolittle RF. Crystal structure of a native chicken fibrinogen at $5.5 \AA$ resolution. (in preparation).

18) Veklich YI, Gorkun OV, Medved LV, Nieuwenhuizen W, Weisel JW. Carboxyl-terminal portions of the $\alpha$ chains of fibrinogen and fibrin. Localization by electron microscopy and the effects of isolated $\alpha \mathrm{C}$ fragments on polymerization. J Biol Chem 268 : 13577-13585, 1993.

19) Gorkun OV, Veklich YI, Medved LV, Henschen
AH, Weisel JW. Role of the $\alpha \mathrm{C}$ domains of fibrin in clot formation. Biochemistry. 33:6986-6997, 1994.

20) McKee PA, Mattock P, Hill RL. Subunit structure of human fibrinogen, soluble fibrin and cross -linked insoluble fibrin. Proc Natl Acad Sci USA 66 : 738-744, 1997.

21) Doolittle RF. Differences in the clotting of lamprey fibrinogen by lamprey and bovine thrombins. Biochem. J. 94 : 735-741, 1965.

22) Cottrell BA, Doolittle RF. Amino acid sequences of lamprey fibrinopeptides A and B and characterization of the junctions split by lamprey and mammalian thrombins. Biochim Biophys Acta 453 : 426-438, 1976. 